\title{
Enhancement of penetration field in vortex nanocrystals due to Andreev bound states
}

\author{
M. I. Dolz,${ }^{1}$ N. R. Cejas Bolecek, ${ }^{2}$ J. Puig, ${ }^{2}$ H. Pastoriza,${ }^{2}$ G. Nieva, ${ }^{2}$ \\ J. Guimpel, ${ }^{2}$ C.J. van der Beek, ${ }^{3}$ M. Konczykowski, ${ }^{4}$ and Y. Fasano ${ }^{2}$ \\ ${ }^{1}$ Departamento de Física, Universidad Nacional de San Luis and CONICET, San Luis, Argentina \\ ${ }^{2}$ Centro Atómico Bariloche and Instituto Balseiro, CNEA, \\ CONICET and Universidad Nacional de Cuyo, Bariloche, Argentina \\ ${ }^{3}$ Centre de Nanosciences et de Nanotechnologies, CNRS, \\ Université Paris-Sud, Université Paris Saclay, Palaiseau, France \\ ${ }^{4}$ Laboratoire des Solides Irradiés, CEA/DRF/IRAMIS, Ecole Polytechnique, \\ CNRS, Institut Polytechnique de Paris, Palaiseau, France
}

(Dated: October 21, 2019)

\begin{abstract}
We study the penetration field $H_{\mathrm{P}}$ for vortex nanocrystals nucleated in micron-sized samples with edges aligned along the nodal and anti-nodal directions of the d-wave superconducting parameter of $\mathrm{Bi}_{2} \mathrm{Sr}_{2} \mathrm{CaCu}_{2} \mathrm{O}_{8-\delta}$. Here we present evidence that the $H_{\mathrm{P}}$ for nanocrystals nucleated in samples with edges parallel to the nodal direction is larger than for the antinodal case, $\sim 72 \%$ at low temperatures. This finding supports the theoretical proposal that surface Andreev bound states appearing in a sample with edges parallel to the nodal direction would produce an anomalous Meissner current that increases the Bean-Livingston barrier for vortex penetration. This has been detected thanks to the nucleation of vortex nanocrystals with a significant surface-to-volume ratio.

PACS numbers: $74.25 . U v, 74.25 . H a, 74.25 . \mathrm{Dw}$
\end{abstract}

The current need for miniaturization of devices calls for a better understanding on how the thermodynamic, structural and magnetic properties of condensed matter are affected by decreasing the sample size. The components of these devices are made of functional materials as small as nanocrystals with hundred particles or less. The physical properties of nanocrystals of hard condensed matter have been studied both experimentally and theoretically for several systems as metallic nanoparticles, semiconductor nanocrystals and films. 114 Some thermodynamic properties of phase transitions, such as the transition temperature, the entropy and enthalpy jumps, are depleted when reducing the size of components at the nanoscale. 1-4 This is ascribed to a reduction in the average binding energy of the nanocrystal due to the high proportion of particles located at the surface having a lesser binding energy than those of the volume.

The nucleation of nanocrystalline vortex matter in micron-sized superconducting samples opens the possibility of studying this general problem in soft condensed matter systems. [5] 9 Surface effects in vortex nanocrystals affect its thermodynamic, structural and magnetic properties. [10 13] For instance, due to surface barriers, the field at which the first vortex penetrates, $H_{\mathrm{P}}, 14,15$ can be larger than the lower critical field $H_{\mathrm{c} 1}$ (depending only on the penetration depth $\lambda$ and the coherence length $\xi$ of the material). This effect becomes more relevant when the surface-to-volume ratio of the number of vortices in nanocrystals enhances. [8, 11] Surface barriers produce hysteretic behavior in the vortex magnetic response and can be of two types. Geometrical barriers are caused by the extra energy cost for flux entry produced by the local enhancement, close to the sample edge, of the otherwise uniform outer field $H$. [16, 17 .
Bean-Livingston (BL) barriers arise from the competition between the Meissner current pushing the vortex inside the sample versus the attraction of the vortex towards the outer image-vortex. [18, 19] On increasing $\mathrm{H}$, the former term dominates and vortices penetrate the sample.

Theoretical studies predict that nucleating vortices in anisotropic d-wave superconductors with Andreev bound states (ABS) can modify the BL surface barrier, and therefore $H_{\mathrm{P}}$. 20] ABS are surface states localized within a distance $\sim \xi$ from the edge of the samples, 21] of a few nanometers for high- $T_{\mathrm{c}}$ 's. These zero-energy excitations appear if the sample edge is oriented along the nodal direction of the $\mathrm{d}$-wave superconducting parameter and generate an anomalous current running opposite to the supercurrents, namely $\mathbf{J}_{\mathbf{S C}}=2 e|\Psi|^{2}$ $\left(2 e / c m\left(\mathbf{A}_{\mathbf{0}}-\mathbf{A}^{\prime}\right)\right)$. 22 The first term is the regular Meissner current and the second is due to the vector potential of the eventually stable ABS. 23] Therefore, a dependence of $H_{\mathrm{P}}$ on the orientation of the sample edge with the anisotropic d-wave order parameter is expected. 23] The decrease in $J_{S C}$ for samples with edges parallel to the nodal direction produces an enhancement of the BL barrier: the term pushing the vortex inside has a lesser magnitude but the attraction towards the outer anti-vortex remains unaltered.

These surface effects are expected to emerge in the magnetic properties of superconducting samples when dramatically decreasing the size of the vortex crystal. In this work we study vortex nanocrystals with $10^{3}-10^{6}$ vortices and 10 to $1 \%$ surface-to-volume ratio nucleated in micron-sized $\mathrm{Bi}_{2} \mathrm{Sr}_{2} \mathrm{CaCu}_{2} \mathrm{O}_{8-\delta}$ cuboids. We study two sets of cuboids, with edges aligned along the nodal $(\mathrm{N})$ and the anti-nodal (AN) directions of the d-wave order parameter. We found that $H_{\mathrm{P}}$ is enhanced in $\mathrm{N}$ with re- 


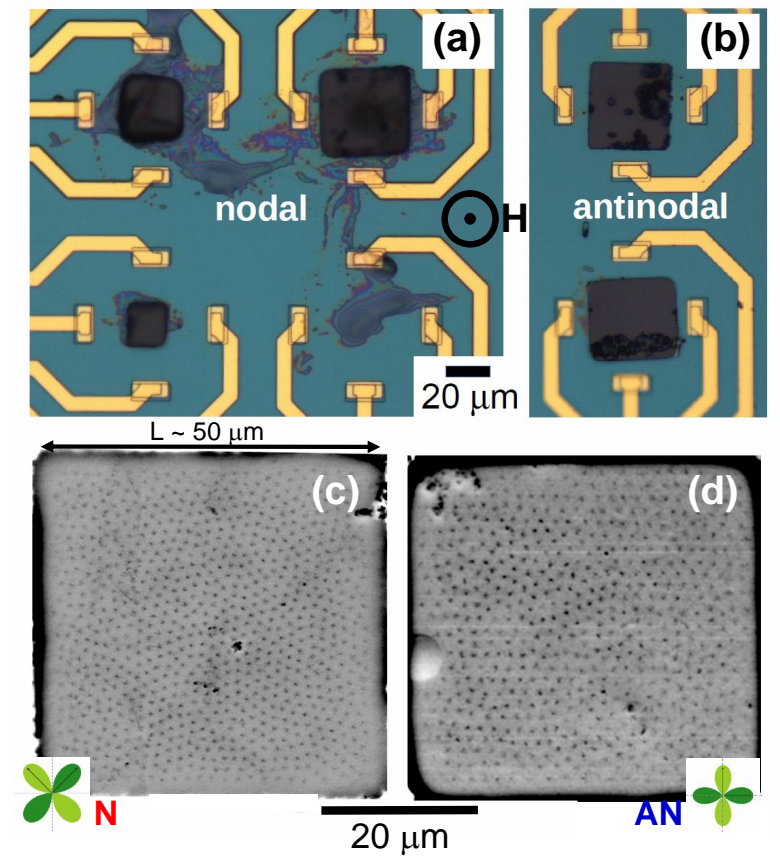

Figure 1: Some of the nodal $(\mathrm{N})$ and antinodal (AN) $\mathrm{Bi}_{2} \mathrm{Sr}_{2} \mathrm{CaCu}_{2} \mathrm{O}_{8+\delta}$ cuboids studied located on top of $16 \times$ $16 \mu \mathrm{m}^{2}$ Hall sensors. (a) N cuboids with sides $L=20,30$ and $50 \mu \mathrm{m}$; (b) AN cuboids with $L \sim 50 \mu \mathrm{m}$. Vortex nanocrystals imaged by magnetic decoration in (c) N (8.5 Gauss) and (d) AN (5 Gauss) cuboids and alignment of the sample edge with the nodes of the d-wave superconducting parameter.

spect to AN cuboids of the same size, up to $~ 72 \%$ at low temperatures. This result has been detected thanks to the virtuous combination of low-noise local magnetic techniques and the nucleation of vortex nanocrystals.

We micro-engineer the cuboids from optimally-doped $\mathrm{Bi}_{2} \mathrm{Sr}_{2} \mathrm{CaCu}_{2} \mathrm{O}_{8}$ macroscopic single-crystals 24] with critical temperature $T_{\mathrm{c}}=90 \mathrm{~K}$. We start from rectangular macroscopic samples aligned with their edges parallel to the $a$ (or $b$ ) crystalline direction, i.e. parallel to the $\mathrm{N}$ direction of the d-wave order parameter. Cuboids were fabricated by combining optical lithography and physical ion milling. 9]. N cuboids are obtained by aligning the optical-lithography mask such that their sides result parallel to the macroscopic sample edge. In a similar fashion, AN cuboids were fabricated rotating the lithography mask $45^{\circ}$ from the previous configuration. [25] We studied several $\mathrm{N}$ and AN cuboids with square sides $L=20$, 30 and $50 \mu \mathrm{m}$, thicknesses $t \sim 2 \mu \mathrm{m}$. The critical temperature of all studied cuboids is the same than that of the parent macroscopic crystal, $T_{\mathrm{c}}=90 \mathrm{~K}$.

Figure 1 shows various samples placed on top of Hallsensors with $16 \times 16 \mu \mathrm{m}^{2}$ working areas micro-fabricated from GaAs/AlGaAs heterostructures. Samples were micro-manipulated and glued with Apiezon $\mathrm{N}$ grease to improve thermal contact. Local ac and dc magnetization measurements were performed applying dc, $H$, and rip- ple, $h_{\text {ac }} \ll H$, magnetic fields parallel to the $c$-axis. The Hall sensor signal is proportional to the magnetization of the cuboids, $H_{\mathrm{s}}=(B-H)$. We measure dc and ac hysteresis loops recording $H_{\mathrm{s}}$ and the sample transmittivity $T^{\prime}$ on sweeping $H . T^{\prime}$ is obtained applying an $h_{\mathrm{ac}}$ and normalizing the in-phase component of the first-harmonic signal, $B^{\prime}$. This magnitude is measured by means of a digital-signal-processing lock-in technique using the lockin reference signal as supply to the coil generating $h_{\mathrm{ac}}$. The normalization $T^{\prime}=\left[B^{\prime}(T)-B^{\prime}\left(T \ll T_{\mathrm{c}}\right)\right] /\left[B^{\prime}(T>\right.$ $\left.\left.T_{\mathrm{c}}\right)-B^{\prime}\left(T \ll T_{\mathrm{c}}\right)\right]$ is such that $T^{\prime}=1$ in the normal state and $T^{\prime}=0$ well within the superconducting phase.

The superconducting quality of the cuboids was checked by magnetic decoration experiments. [26] Figure 1 (c) and (d) show vortex nanocrystals nucleated at low vortex densities in $\mathrm{N}$ and $\mathrm{AN}$ cuboids with $L=$ $50 \mu \mathrm{m}$. Black dots correspond to individual vortices decorated with magnetized Fe nanoparticles attracted to the cores due to their local field gradient. Regular vortex structures with an excess of topological defects induced by confinement [13] are observed for both, $\mathrm{N}$ and $\mathrm{AN}$ cuboids.

Figure 2 (a) shows illustrative dc hysteresis loops at $54 \mathrm{~K}$ in $\mathrm{N}$ and AN cuboids, both with $L=50 \mu \mathrm{m}$. On increasing field from zero, $H_{\mathrm{s}}$ follows first the linear Meissner response associated to complete field expulsion. The entrance of the first vortex into the cuboids is signposted by the departure of $H_{\mathrm{s}}$ from linearity, at a field $H_{\mathrm{p}}$. On further increasing field, $H_{\mathrm{s}}$ changes curvature as expected when vortices penetrate. The dc loops have a two-quadrant locus, with branches located mostly in the second and forth quadrant. The two field-descending branches for positive and negative $H$ are almost horizontal and close to zero. The same behavior is observed for all dc loops measured between 35 and $90 \mathrm{~K}$. 225] This phenomenology suggests that for these vortex nanocrystals bulk pinning has a lesser effect than surface barriers for vortex penetration.

We also measured $H_{\mathrm{p}}$ in $\mathrm{N}$ and $\mathrm{AN}$ cuboids from ac hysteresis loops, see Fig. 2 (b). At low fields $T^{\prime}=0$, indicating full expulsion of the magnetic field; on increasing field, $T^{\prime}$ becomes non-negligible at roughly the same $H_{\mathrm{p}}$ where the departure of linearity is detected in dc loops, see dashed line in Fig.2 On further increasing $H$ the number of vortices penetrating the sample enhances and $T^{\prime}$ grows in accordance, reaching a value close to 1 for high fields. For a given $H$ in the same loop, $T^{\prime}$ in the descending branch is larger than in the ascending one since in the former there is extra trapped flux.

The most remarkable result of dc and ac data of Fig.2 is that the penetration field for $\mathrm{N}$ is larger than for AN cuboids. Figure 3 (a) shows a comparison between the temperature-evolution of $H_{\mathrm{p}}$ for $\mathrm{N}$ and AN cuboids with $L=50 \mu \mathrm{m}$. The values obtained considering the virgin branches of ac (open points) and dc (full points) magnetization loops are similar within the uncertainty. In all 


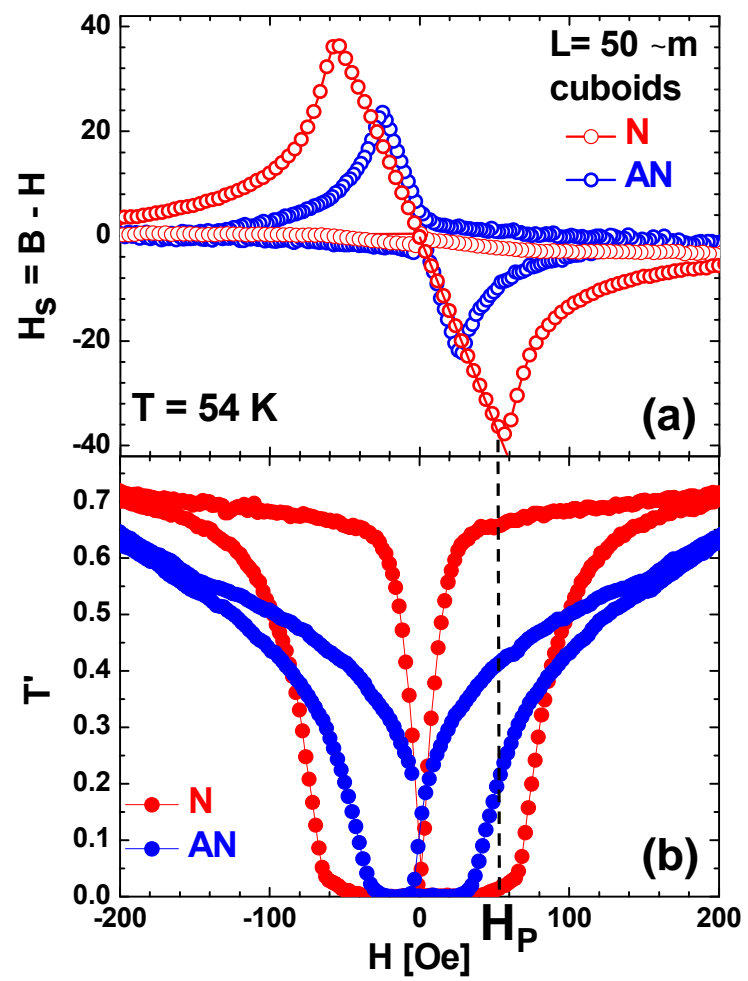

Figure 2: Detection of the penetration field $H_{\mathrm{p}}$ in nodal $(\mathrm{N})$ and antinodal (AN) cuboids (with $L=50 \mu \mathrm{m}$ ) from (a) dc and (b) ac magnetic hysteresis loops. The $H_{\mathrm{p}}$ determined from the deviation of the linear Meissner response coincides with the field at which transmittivity becomes non-negligible (see vertical dashed lines). Measurements performed at $54 \mathrm{~K}$; ac measurements with $h_{\mathrm{ac}}$ of 1 Gauss and $7.1 \mathrm{~Hz}$.

the studied temperature-range, $H_{\mathrm{p}}^{\mathrm{N}}$ is larger than $H_{\mathrm{p}}^{\mathrm{AN}}$ beyond error bars, with this difference increasing on cooling. For instance, for the smallest measured temperature, $T / T_{\mathrm{c}} \sim 0.4, H_{\mathrm{p}}^{\mathrm{N}} \simeq 1.72 H_{\mathrm{p}}^{\mathrm{AN}}$.

Even though $H_{\mathrm{p}}$ is sensitive to the side of the cuboids, this difference can not be accounted by eventual small changes in $L$. Figure 4 shows the temperaturedependence of $H_{\mathrm{p}}$ for $\mathrm{N}$ cuboids with $L=20,30$ and $50 \mu \mathrm{m}$. The smaller the $L$, the larger $H_{\mathrm{p}}$, but the separation between curves enlarge on decreasing $T$. In particular, at $T / T_{\mathrm{c}} \sim 0.4$ the $H_{\mathrm{p}}$ difference between the 20 and $50 \mu \mathrm{m}$ cuboids is of $\sim 15 \%$, much smaller than that found between $H_{\mathrm{p}}^{\mathrm{AN}}$ and $H_{\mathrm{p}}^{\mathrm{N}}$ at the same $T$.

We calculated the ratio $H_{\mathrm{p}}^{\mathrm{N}} / H_{\mathrm{p}}^{\mathrm{AN}}$ by averaging $H_{\mathrm{p}}$ data obtained from ac and dc measurements, see Fig. 3 (b). This ratio seems to be featureless in temperature, even if the error bars are significant. The figure also shows the theoretically-expected evolution of $H_{\mathrm{p}}^{\mathrm{N}} / H_{\mathrm{p}}^{\mathrm{AN}}$ when considering the effect of Andreev bound states in the BL surface barrier for an ideal infinite sample of a d-wave superconductor with $\kappa=\lambda / \xi=200$, and neglecting any other barrier for vortex penetration. 23. For the vortex nanocrystals studied here, $\kappa \sim 200$ as in the the-

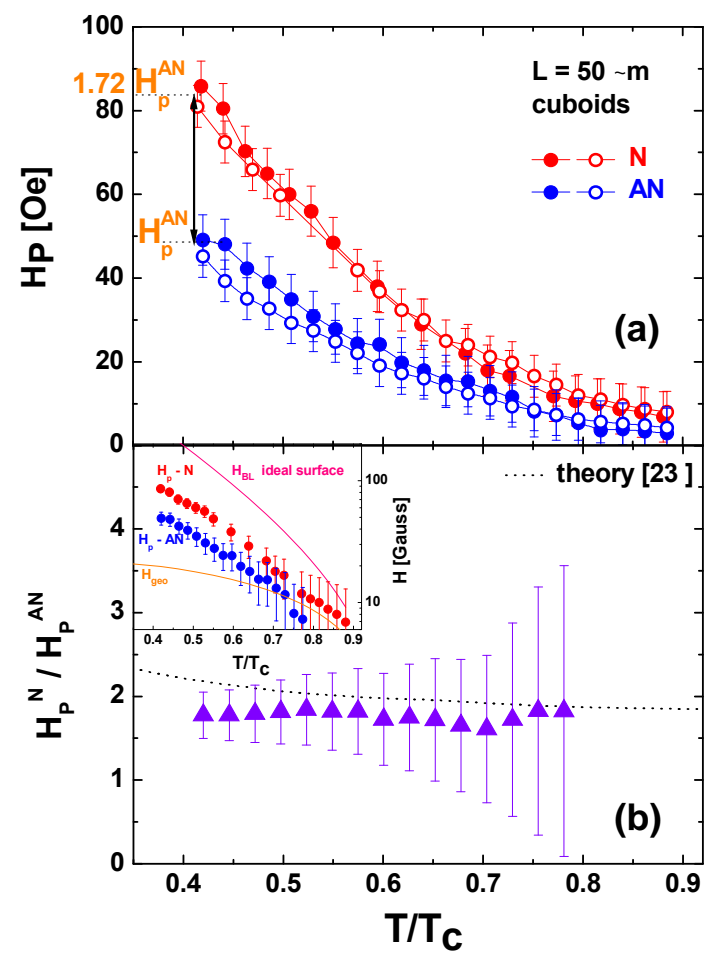

Figure 3: (a) Temperature-dependence of the penetration field $H_{\mathrm{p}}$ for nodal $(\mathrm{N})$ and antinodal $(\mathrm{AN})$ cuboids from dc and ac (open and full symbols) measurements. (b) $H_{\mathrm{p}}^{\mathrm{N}} / H_{\mathrm{p}}^{\mathrm{AN}}$ data (symbols) and temperature-dependence predicted in Ref.23] (black dotted line) for a superconductor with $\kappa=200$. Insert: Data of (a) compared with the theoretical expectation for geometrical (orange) and BL (pink) barriers for cuboids of the same size and material than we study.

oretical study, but the cuboids are not an ideal infinite interface. However, our $\mathrm{N}$ and AN experimental data are quite close to the theoretically-expected BL barrier for an isotropic superconducting cuboid with $L=50 \mu \mathrm{m}$ and $t=2 \mu \mathrm{m}$, see pink curve in the insert. This curve was obtained considering that the temperaturedependence of $H_{\mathrm{p}}$ for a BL barrier in an ideally specular surface is $H_{\mathrm{BL}}(T)=H_{\mathrm{c}}(T) \sqrt{t / L} \exp \left(-T / T_{0}\right)$. $H_{\mathrm{c}}(T) \simeq \kappa H_{\mathrm{c} 1}(T) / \ln \kappa$ is the thermodynamic critical field with $H_{\mathrm{c} 1}(T)$ the first critical field, and $T_{0} \sim 15 \mathrm{~K}$ for $\mathrm{Bi}_{2} \mathrm{Sr}_{2} \mathrm{CaCu}_{2} \mathrm{O}_{8}$ [8], a characteristic temperature below which pancake vortices are individually pinned. [8, 19] This ideal $H_{\mathrm{BL}}(T)$ coincides with the data at high $T$, and overpass them by more than a factor two at low $T$. This discrepancy quite likely has origin in the non-ideal nature of the edges of our samples.

Nevertheless, the $H_{\mathrm{p}}^{\mathrm{N}} / H_{\mathrm{p}}^{\mathrm{AN}}$ experimental data lay pretty close to the theoretical value expected when considering the effect of Andreev bound states, even though this theoretical curve has no other free parameter than the $\kappa$ of the superconducting material. In addition, geometrical barriers do not seem to play a relevant role at $T / T_{\mathrm{c}} \lesssim 0.6$ as to explain the enhancement of $H_{\mathrm{p}}^{\mathrm{N}}$ versus 


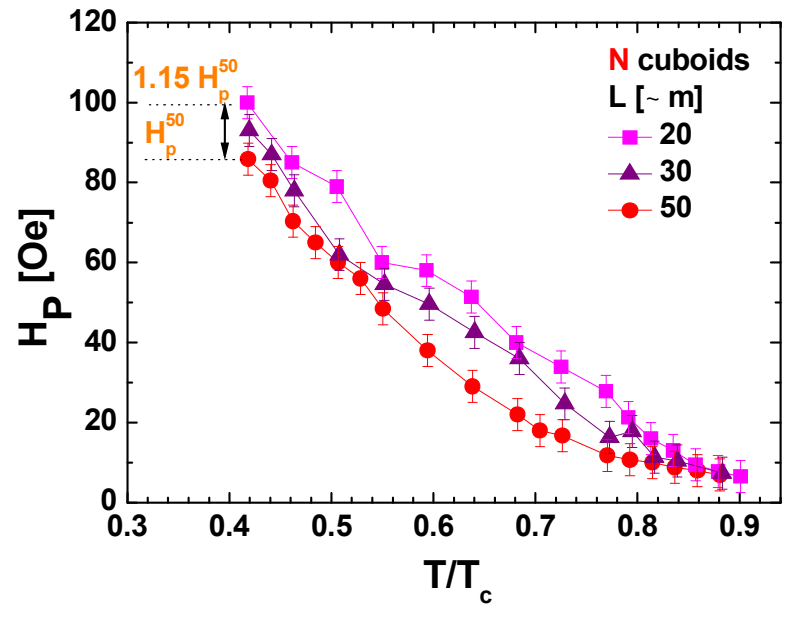

Figure 4: Temperature-dependence of the penetration field for nodal cuboids with different square sides of $L=20,30$ and $50 \mu \mathrm{m}$. Data from ac magnetization in agreement with dc data within the error. Measurements performed with a ripple field of 1 Gauss and $7.1 \mathrm{~Hz}$.

that of $H_{\mathrm{p}}^{\mathrm{AN}}$. Indeed, the insert to Fig.3 (b) shows that $H_{\text {geo }}$, the penetration field associated to the geometrical barrier for the geometry of the studied cuboids is always below the experimental $\mathrm{N}$ and $\mathrm{AN} H_{\mathrm{p}}$. This curve is obtained considering that $H_{\text {geo }}=H_{\mathrm{c} 1}(T) \tanh \sqrt{0.36 t / L}$ with 0.36 the geometrical factor for cuboids. 27]

In conclusion, the comparison between the theoretical BL and geometrical barriers in cuboids, and our experimental data in cuboids of a d-wave superconductor, suggests the dominance of the BL barrier for vortex penetration at low $T$. This can be at the origin of the rather good quantitative agreement between the experimental data and the theoretically predicted $H_{\mathrm{p}}^{\mathrm{N}} / H_{\mathrm{p}}^{\mathrm{AN}}$ for d-wave ideal infinite superconductors, a striking result considering that this theoretical calculation depends only in the value of $\xi$ for the superconducting material. The latter suggests that the barriers for vortex penetration in $\mathrm{N}$ cuboids are fully governed by the decrease in the effective Meissner current induced by the presence of Andreev bound states located only up to a distance $\xi \sim 5 \cdot 10^{-4} L$ from the surface of the cuboids. Therefore, we present here evidence of a new phenomena in the magnetic properties of vortex nanocrystals emergent from the microscopic local electronic properties of anisotropic dwave superconductors. From an applied point of view, our data suggests that the crystal orientation of micronsized superconducting samples made of high- $T_{\mathrm{c}}$ 's, with quite frequently anisotropic order parameters, is a property that has to be taken into account for the magnetic response of devices based in such tiny building blocks.

We thank V. Mosser for providing the sensors, M. $\mathrm{Li}$ for growing the macroscopic crystals, the ECOSSud Argentina-France collaboration and grants ANR-10LABX-0039-PALM and ANR-CE08-007 DiSSCo-Hall for financial support.

[1] C.J. Coombes, J. Phys. F: Metal Phys. 2, 441 (1972).

[2] A. N. Goldstein, C. M. Echer, and A. P. Alivisatos, Science 256, 1425 (1992).

[3] S. H. Tolbert and A.P. Alivisatos, Science 265, 373 (1994).

[4] G.Guisbiers and L.Buchaillot, Phys. Lett. A 374, 305(2009).

[5] V. V. Moshchakov, L. Gielen, C. Strunk, R. Jonckheere, X. Qiu, C. Van Haesendonck and Y. Bruynseraede, Nature 373, 319 (1995).

[6] A. K. Geim, I. V. Grigorieva, S. V. Dubonos, J. G. S. Lok, J. C. Maan, A. E. Filippov and F. M. Peeters, Nature 390, 259 (1997).

[7] V. A. Schweigert, F. M. Peeters, P. S. Deo, Phys. Rev. Lett. 812783 (1998).

[8] Y.M. Wang, M.S. Fuhrer, A. Zettl, S. Ooi, T. Tamegai, Phys. Rev. Lett. 86, 3626 (2001).

[9] M.I. Dolz, Y. Fasano, N.R. Cejas Bolecek, H. Pastoriza, V. Mosser, M. Li and M. Konczykowski, Phys. Rev. Lett. 115, 137003 (2015).

[10] J. J. Palacios, Phys. Rev. B 58, R5948 (1998).

[11] M. I. Dolz, Y. Fasano, N. R. Cejas Bolecek, H. Pastoriza, M. Konczykowski and C. J. van der Beek, Journal of Physics: Conference Series 568, 022010 (2014).

[12] N. R. Cejas Bolecek, M. I. Dolz, A. Kolton, H. Pastoriza, C. J. van der Beek, M. Konczykowski, M. Menghini, G. Nieva and Y. Fasano, Jounal Low Temp. Phys. 17935 (2015).

[13] N. R. Cejas Bolecek, M. I. Dolz, H. Pastoriza, M. Konczykowski, C. J. van Der Beek, A. B. Kolton and Y. Fasano, Phys. Rev. B 96, 024507 (2017).

[14] M. Konczykowski, L. I. Burlachkov, Y. Yeshurun, and F. Holtzberg, Phys. Rev. B 43, R13707 (1991).

[15] J.R. Clem, Journal of Supercond. and Novel Magn. 6, 1557 (2008).

[16] E. Zeldov, A. I. Larkin, V. B. Geshkenbein, M. Konczykowski, D. Majer, B. Khaykovich, V. M. Vinokur and H. Shtrikman, Phys. Rev. Lett. 73, 1428 (1994).

[17] R. Willa, V. B. Geshkenbein and G. Blatter, Phys. Rev. B 89, 104514 (2014).

[18] A. E. Koshelev, Physica C 191, 219 (1992).

[19] L. Burlachkov, Phys. Rev. B 47, 8056 (1993).

[20] M. Fogelström, D. Rainer, and J. A. Sauls, Phys. Rev. Lett. 79, 281 (1997).

[21] C-R. Hu, Phys. Rev. Lett. 72, 1526(1994).

[22] C. Iniotakis, S. Graser, T. Dahm and N. Schopohl, Phys. Rev. B 71, 214508 (2005).

[23] C. Iniotakis, T. Dahm and N. Schopohl, Phys. Rev. Lett. 100, 037002 (2008).

[24] T. W. Li, P.H. Kes, N.T. Hien, J. J. M. Franse and A. A. Menovsky, J. Cryst. Growth 135, 481 (1994).

[25] See Supplemental Material at [URL will be inserted by publisher] for further details in the preparation of $\mathrm{N}$ and AN cuboids, as well as temperature-evolution of dc magnetization loops.

[26] Y. Fasano, J. Herbsommer, and F. de la Cruz, Phys. Stat. Sol. (b) 215, 563 (1999).

[27] E. H. Brandt, Phys. Rev. B 60, 11939 (1999). 